\title{
LAS TIC EN LAS COMUNICACIONES EN LA ESCUELA. PERCEPCIÓN DE PROFESORADO Y FAMILIAS
}

\author{
Elena Briones \\ Área de Psicología Evolutiva y de la Educación \\ Universidad de Cantabria. Facultad de Educación. \\ brionese@unican.es \\ Marta García Lastra \\ Área de Sociología \\ Universidad de Cantabria. Facultad de Educación. \\ Eva M. Gómez-Pérez \\ Área de Psicología Evolutiva y de la Educación \\ Universidad de Cantabria. Facultad de Educación.
}

Recepción Artículo: 10 febrero 2020

Admisión Evaluación: 4 marzo 2020

Informe Evaluador 1: 13 marzo

Informe Evaluador 2: 17 marzo 2020

Aprobación Publicación: 20 abril 2020

\section{RESUMEN}

En este trabajo se aborda una de las consecuencias de la llegada de las TIC al mundo educativo menos estudiada hasta el momento: Ios cambios que estas están produciendo en las relaciones familia-escuela al promover nuevas formas de comunicación 0, de manera paralela, fórmulas diversas para aumentar su implicación y participación. En concreto, se analiza la percepción que familias y profesorado de distintas etapas educativas tienen de las TIC en sus comunicaciones, atendiendo a su uso, adecuación y temáticas abordadas. Así mismo, se indaga en la relación entre la percepción de las relaciones en la comunidad educativa, la valoración de las TIC y temáticas abordadas en las comunicaciones.

Los resultados evidencian diferencias en función de la etapa analizada, así como también ciertas similitudes y concordancias en las percepciones de familias y profesorado. De esta manera, se identifica que la comunicación en Educación Secundaria se concentra en el control y seguimiento en mayor medida que en Primaria, nivel en el que el intercambio de información sobre actividades extraescolares, materiales o distintos aspectos del centro es una temática habitual a la que el profesorado dedica más atención. Los medios percibidos como menos idóneos son las redes sociales y los mensajes de WhatsApp. Se concluye valorando la necesidad de fomentar la participación en el centro escolar reforzando el uso adecuado de las TIC, con el fin último de favorecer el abordaje de temáticas que vayan más allá del control y seguimiento escolar.

Palabras clave: TIC; comunicación educativa; relaciones entre la comunidad educativa; profesorado; familias 


\section{LAS TIC EN LAS COMUNICACIONES EN LA ESCUELA. PERCEPCIÓN DE PROFESORADO Y FAMILIAS}

\section{ABSTRACT}

The twitches in school communications. Teachers' and families' perception. This paper is focused on one of the less studied consequences of the arrival of ICT to educational world: the changes are producing in family-school relationships due to new forms of communication or, in parallel, different ways to enlarge their involvement and participation. Particularly, it is analyzed the perception that families and teachers of different educational stages have about ICT in their communications, according to their use, adequacy and themes addressed. It also investigates whether the perception of the relationship between the educational community is related to the perception of the suitability of ICT in communications.

Data show differences depending on the stage analyzed, as well as certain similarities and concordances in the perceptions of families and teachers. In this way, it identifies that the communication in secondary education is more concentrated on the control and monitor than in primary, in which the exchange of information on extracurricular activities, materials or different aspects of the center are the common subjects teachers devote more attention. Tools perceived as less suitable are social networks and WhatsApp. It is concluded by evaluating the need to promote the participation at school by reinforcing the proper use of ICT, in order to favor the treatment of topics that go beyond control and monitoring.

Keywords: ICT; educational communication; relationship between the educational community; teachers; families

\section{INTRODUCCIÓN}

La llegada de las TIC a la escuela es una realidad que ha corrido pareja a la introducción de estas en otros ámbitos de nuestras sociedades como requisito, y consecuencia, de la Sociedad de la Información y la Comunicación. En un breve espacio de tiempo, las TIC se han convertido en una pieza más en su funcionamiento cotidiano: han modificado las formas de enseñar, de aprender (Engell, Coll y Bustos, 2010; Fernández Prados, 2012; Fernández Enguita y Vázquez Cupeiro, 2017) y, además, han transformado la forma de comunicarse entre la escuela y las familias, una nueva situación que es vista de un lado como una forma de promover nuevas formas de participación e implicación de estas últimas (Vázquez López y Colmenares, 2014), 0, de manera menos ambiciosa, como un nuevo canal de información (Sánchez-Garrote y Cortada-Pujol, 2015).

Diversos estudios realizados (OImstead, 2013; Bernstein, 1998, citado por Maciá y Garreta, 2018), han demostrado cómo esta nueva forma de comunicarse ha repercutido en su eficiencia, eficacia, rapidez, efectividad, conveniencia, y, además, ha conseguido aliviar una de las dificultades habitualmente señaladas a la hora de hablar de los problemas en las relaciones entre familia y escuela: a saber, la incompatibilidad de tiempos que en este nuevo escenario es superada al romper los espacios físicos y temporales para su encuentro. En este sentido, las TIC deben ser consideradas un instrumento para la mejora de estas relaciones demandada por las propias familias (García Sanz, Gomariz, Hernández Prados y Parra, 2010). No sin olvidar las dificultades que pueden surgir derivadas de problemas de acceso 0 utilización adecuada de las TIC por parte de las familias, al tiempo de las actitudes hacia su uso en este contexto (Maciá y Garreta, 2018).

En este estudio se aborda el análisis de la percepción de docentes y familias del uso de las TIC en las comunicaciones entre familia y escuela (recursos, idoneidad y temáticas); así como de la percepción de docentes y familias de sus relaciones, atendiendo a las posibles diferencias según etapa educativa. Además, se indaga la relación entre la percepción de las relaciones entre familia y escuela, y la valoración de las TIC y temáticas abordadas. En este sentido, se espera una relación positiva entre la percepción de las relaciones entre la comunidad educativa y la valoración de las TIC, así como con la libertad para abordar diversas temáticas.

\section{METODOLOGÍA}

\section{Muestra}

La población del estudio son las familias y el profesorado del alumnado escolarizado durante el curso 2016- 
2017 en Cantabria. El proceso de muestreo fue aleatorio por conglomerados bietápico (i.e., selección aleatoria de centros educativos y aulas) con carácter estratificado según curso, titularidad del centro (i.e., público, concertado-privado) y tipo de municipio (i.e., rural, intermedio y urbano). La muestra de familias se obtuvo mediante muestreo aleatorio entre las familias de los estudiantes de las aulas finalmente seleccionadas. La participación del profesorado se solicitó online en la plataforma institucional. La muestra quedó integrada por 511 familias, 309 de estudiantes de Primaria (16.6\% padres, 82\% madres, 1,3 otros), y 146 de estudiantes de ES0 (19.1\% padres, $80.1 \%$ madre, $0.7 \%$ otros); y la muestra del profesorado por 158 profesores/as de Primaria, Secundaria, además de Infantil.

\section{Diseño}

El diseño es transversal, descriptivo y correlacional pues permite obtener información para responder a los objetivos del estudio.

\section{Variables e instrumentos}

A continuación, se describen los instrumentos de la medición de cada variable según grupo de informante.

Familias:

Percepción de las relaciones con el personal del centro educativo. Esta medida comprende tres ítems para valorar la calidad de la relación con el equipo directivo, el profesorado y el/la tutor/a de su hijo, empleando una escala Likert de 5 puntos (1, "Muy mala"; 5 , "Muy buena"). La fiabilidad es de

\section{Profesorado:}

Percepción de las relaciones interpersonales de la comunidad escolar (Cuestionario de García y Castro, 2013). Se emplea la escala sobre las relaciones entre profesorado y familias de 6 ítems $(\boldsymbol{V}=.73)$, con escala de respuesta Likert de 4 puntos (1, "Nada"; 4, "Mucho"). Además, se incorporan dos preguntas ("Las familias participan en la organización de actividades en el centro"; "¿Se potencia la participación de las familias de alguna otra forma?"). La fiabilidad con los 8 ítems es de .81. Se construye una medida final, y otras tres con sus factores: participación de las familias (3 ítems, $\boldsymbol{V}=.78$ ), fomento de la participación familiar (2 ítems, $\boldsymbol{V}=.53$ ) y competencia profesional auto-percibida en la comunicación y gestión de conflictos (3 ítems, $\boldsymbol{V}=.78$ ).

Percepción de dificultades en la relación con las familias. Se mide con el ítem: "Existen dificultades en la relación con las familias?", empleando una escala de respuesta Likert de 4 puntos (1, "Nada"; 4, "Mucho").

Familias y Profesorado:

Uso y adecuación de TIC en la comunicación entre familia y centro escolar. Se propone la valoración de: "página web, blogs, redes sociales, email, SMS, WhatsApp, otro/s". La escala de respuesta presenta la opción de O ("no se utiliza"), y una escala Likert de 4 puntos (1, "Nada adecuada", 4 "Muy adecuada") para valorar su adecuación.

Medio más utilizado para la comunicación con las familias. Se plantea la pregunta abierta: “¿Cuál es el medio más utilizado para comunicarse con las familias?' .

Contenidos de las comunicaciones mantenidas mediante TIC. Se solicita la frecuencia (1, "Nunca"; 2, "SÍ, algunas veces"; 3 , "Sí, con frecuencia) con la que se abordan 13 temáticas (García Sanz et al., 2010) y se ofrece la cuestión abierta "otros contenidos". Estas temáticas se factorizan en: Control y seguimiento (4 ítems; $\boldsymbol{V}=.69$, en ambas muestras); Participación (4 ítems; $\boldsymbol{V}=.75$ y .65); Información (3 ítems; $\boldsymbol{V}=.72$ y .73); Desarrollo personal y adaptación (2 ítems; $\boldsymbol{V}=.87$ y .80, en las muestras de familias y profesores, respectivamente).

\section{Procedimiento}

Una vez contactados los centros educativos seleccionados, se solicitó la colaboración del equipo directivo y 


\section{LAS TIC EN LAS COMUNICACIONES EN LA ESCUELA. PERCEPCIÓN DE PROFESORADO Y FAMILIAS}

profesorado para acceder a las familias. Estas cumplimentaron de forma anónima y voluntaria el cuestionario, una vez informadas del objetivo del estudio. Se siguió el mismo procedimiento con el profesorado que realizó el cuestionario online.

Se han salvaguardado las cuestiones éticas, siguiendo el Código de Buenas Prácticas del CSIC (2011). El estudio cuenta con la aprobación del Comité de Ética de la Investigación en Humanidades y Ciencias Sociales de

la Universidad de Cantabria.

\section{Análisis de datos}

LoS análisis cuantitativos se han realizado empleando el programa SPSS 15.0. Los cualitativos consisten en el análisis de contenido para identificar categorías emergentes, siguiendo un proceso inductivo y la triangulación de investigadores (Ruiz, 2003).

\section{RESULTADOS}

Percepciones de las familias

Se presentan las correlaciones entre las variables cuantitativas del estudio (Tabla 1).

Tabla 1

Correlaciones entre las variables cuantitativas de la muestra de familias.

\begin{tabular}{|c|c|c|c|c|c|c|c|c|c|c|c|c|}
\hline $\begin{array}{l}\text { Variabl } \\
\text { es }\end{array}$ & 1 & 2 & 3 & 4 & 5 & 6 & 7 & 8 & 9 & 10 & 11 & 12 \\
\hline $\begin{array}{l}\text { 1.Rel. } \\
\text { Dir }\end{array}$ & & & & & & & & & & & & \\
\hline $\begin{array}{l}\text { 2.Rel.P } \\
\text { rof }\end{array}$ & $.53_{*}^{* *}$ & & & & & & & & & & & \\
\hline $\begin{array}{l}\text { 3.Rel.T } \\
\text { ut. }\end{array}$ & $.37_{*}^{* *}$ & $.57^{* *}$ & & & & & & & & & & \\
\hline $\begin{array}{l}\text { 4.Contr } \\
\text { ol }\end{array}$ & .06 & .02 & .03 & & & & & & & & & \\
\hline $\begin{array}{l}\text { 5.Partic } \\
\text { ip. }\end{array}$ & $.11^{*}$ & .06 & .05 & $.64^{*}$ & & & & & & & & \\
\hline $\begin{array}{l}\text { 6.Infor } \\
\text { m. }\end{array}$ & .03 & .00 & -.04 & $.48^{*}$ & $.66^{*}$ & & & & & & & \\
\hline $\begin{array}{l}\text { 7.DesP } \\
\text { er. }\end{array}$ & $.13^{* *}$ & .05 & .07 & $.54^{*}$ & $.66_{* *}^{*}$ & $.45^{*}$ & & & & & & \\
\hline $\begin{array}{l}\text { 8.Pag } \\
\text { Web }\end{array}$ & .07 & .07 & .05 & $.17^{* *}$ & $.17^{*}$ & $.11^{*}$ & .12 & & & & & \\
\hline 9.Blogs & .08 & $.12^{*}$ & .07 & $.15^{*}$ & $.17^{*}$ & .08 & .17 & $.45^{*}$ & & & & \\
\hline $\begin{array}{l}10 . \text { Red } \\
\text { SS }\end{array}$ & .02 & -.03 & -.05 & $.15^{*}$ & $.18^{* *}$ & .10 & .17 & $.32^{*}$ & $.36^{*}$ & & & \\
\hline $\begin{array}{l}\text { 11.Ema } \\
\text { il }\end{array}$ & .00 & -.02 & -.03 & $.14^{*}$ & $.18^{* *}$ & $.11^{*}$ & .13 & $.40_{* *}^{*}$ & $.29^{*}$ & $.58^{*}$ & & \\
\hline $\begin{array}{l}\text { 12.SM } \\
\mathrm{S}\end{array}$ & -.01 & -.07 & $.15^{* *}$ & .03 & $.10^{*}$ & .01 & .09 & $.24^{*}$ & $.20_{* *}^{*}$ & $.52^{*}$ & $.58^{*}$ & \\
\hline $\begin{array}{l}\text { 13.Wh } \\
\text { ats. }\end{array}$ & .03 & -.01 & -.03 & .09 & $.13^{*}$ & .05 & .17 & $.20_{* *}^{*}$ & $.28^{*}$ & $.54^{*}$ & $.52^{*}$ & $.66_{* *}^{*}$ \\
\hline
\end{tabular}

Nota: $* * * p<.001 ; * * p<.01 ; * p<.05$ 
Los resultados de los ANOVAS considerando como factor la etapa educativa informan de que las familias con hijos en la etapa de Primaria perciben más positivas las relaciones con la dirección, el/la tutor/a y el profesorado del centro educativo que en la etapa de Secundaria (Tabla 2).

Tabla 2

Descriptivos en la percepción de las familias de la relación con la dirección, profesorado y tutor/a del centro educativo de su hijo/a, según etapa educativa.

\begin{tabular}{llccc}
\hline \multicolumn{1}{c}{ Variables dependientes } & $\begin{array}{c}\text { Niveles del } \\
\text { factor }\end{array}$ & Media & DT & Prueba F \\
\hline \multirow{3}{*}{ Relación con la Dirección } & Primaria & 4.39 & 0.73 & \\
& Secundaria & 4.22 & 0.73 & $\mathrm{~F}(1,436)=5.26^{*}$ \\
& Total & 4.34 & 0.73 & \\
\hline \multirow{2}{*}{ Relación con el } & Primaria & 4.59 & 0.63 & \\
Profesorado & Secundaria & 4.26 & 0.69 & $\mathrm{~F}(1,437)=524.57^{* *}$ \\
& Total & 4.49 & 0.66 & \\
\hline \multirow{3}{*}{ Relación con el Tutor } & Primaria & 4.59 & 0.63 & \\
& Secundaria & 4.47 & 0.62 & $\mathrm{~F}(1,435)=3.69^{*}$ \\
& Total & 4.55 & 0.63 & \\
\hline
\end{tabular}

Nota: $* * p<.01 ; * p<.05$

Además, en la percepción de las familias sobre las TIC empleadas, se observan diferencias significativas en la percepción de la idoneidad del email y del SMS, pues son más valorados por las familias que tienen hijos/as en la etapa de Secundaria. Por otra parte, los medios percibidos como menos idóneos son el WhatsApp y las redes sociales, en ambas etapas (Tabla 3).

Tabla 3

Percepción de las familias del uso de las TIC y su adecuación para la comunicación entre la familia y el centro según etapa educativa.

\begin{tabular}{|c|c|c|c|c|c|}
\hline & \multicolumn{4}{|c|}{ Etapa Educativa } & \multirow[b]{3}{*}{ Prueba F } \\
\hline & \multicolumn{2}{|c|}{ Primaria } & \multicolumn{2}{|c|}{ Secundaria } & \\
\hline & $\begin{array}{c}\% \text { No se } \\
\text { utiliza }\end{array}$ & Media/DT & $\begin{array}{c}\% \text { No se } \\
\text { utiliza }\end{array}$ & Media/DT & \\
\hline Página web & $37.6 \%$ & $2.83 / 0.85$ & $15.3 \%$ & $2.89 / 0.78$ & $F(1,286)=0.39$ \\
\hline Blogs & $37.0 \%$ & $2.89 / 0.91$ & $36.5 \%$ & $2.75 / 0.91$ & $F(1,253)=0.79$ \\
\hline Redes sociales & $65.9 \%$ & $2.34 / 0.83$ & $31.7 \%$ & $2.39 / 0.96$ & $F(1,175)=0.17$ \\
\hline Email & $64.2 \%$ & $2.82 / 0.92$ & $24.2 \%$ & $3.14 / 0.82$ & $\mathrm{~F}(1,194)=6.90 * *$ \\
\hline SMS & $80.8 \%$ & $2.38 / 0.93$ & $53.2 \%$ & $3.17 / 0.92$ & $\mathrm{~F}(1,109)=19.85 * * *$ \\
\hline WhatsApp & $73.9 \%$ & $2.67 / 1.15$ & $65.9 \%$ & $2.67 / 1.04$ & $\mathrm{~F}(1,111)=0.21$ \\
\hline Otro/s & $58.3 \%$ & $3.09 / 0.97$ & $47.7 \%$ & $3.13 / 0.97$ & $F(1,75)=0.02$ \\
\hline
\end{tabular}

Nota: $* * * p<.001 ; * * p<.01$

A la pregunta sobre el medio más utilizado por las familias, sólo responde el $5.7 \%$, y se identifica que es el teléfono ( $n=11)$ y la agenda escolar $(n=7)$ en la etapa de Primaria, y la plataforma en ambas etapas $(n=3$, en cada ciclo).

Las pruebas $t$ para muestras relacionadas ( $p<.001)$ permiten ordenar las temáticas según su presencia en las comunicaciones, así, control y seguimiento e información ocupan el primer lugar ( $p=.95)$ seguidos de participa- 


\section{LAS tic EN LAS COMUNICACIONES EN LA ESCUELA. PERCEPCIÓN DE PROFESORAdO Y FAMILIAS}

ción y desarrollo personal ( $p=.95$ ), sin diferencias entre dichos pares. Los ANOVAS con el factor etapa educativa, muestran que las familias cuyos hijos/as están en la etapa de Secundaria perciben en mayor medida que en la etapa anterior, que las comunicaciones con el centro educativo priorizan el control y seguimiento, la participación y el intercambio de información (Tabla 4).

Tabla 4

Descriptivos en las temáticas y resultados de los ANOVAS según etapa educativa de los hijos/as.

\begin{tabular}{|c|c|c|c|c|}
\hline Temáticas de las conversaciones & Etapa & Media & DT & Prueba F \\
\hline \multirow{3}{*}{ Control y Seguimiento } & Primaria & .85 & 0.53 & \multirow{3}{*}{$\begin{array}{c}\mathrm{F}(1,437)=16.07 * * \\
*\end{array}$} \\
\hline & $\begin{array}{l}\text { Secundar } \\
\text { ia }\end{array}$ & 1.08 & 0.61 & \\
\hline & Total & .93 & 0.57 & \\
\hline \multirow{3}{*}{ Participación } & Primaria & .63 & 0.53 & \multirow{3}{*}{$\mathrm{F}(1,430)=7.73 * *$} \\
\hline & $\begin{array}{l}\text { Secundar } \\
\text { ia }\end{array}$ & .79 & 0.59 & \\
\hline & Total & .69 & 0.55 & \\
\hline \multirow{3}{*}{ Información } & Primaria & .87 & 0.64 & \multirow{3}{*}{$\mathrm{F}(1,427)=4.79 *$} \\
\hline & $\begin{array}{l}\text { Secundar } \\
\text { ia }\end{array}$ & 1.02 & 0.64 & \\
\hline & Total & .92 & 0.64 & \\
\hline \multirow{3}{*}{ Desarrollo personal y adaptación } & Primaria & .69 & 0.69 & \multirow{3}{*}{$F(1,423)=0.49$} \\
\hline & $\begin{array}{l}\text { Secundar } \\
\text { ia }\end{array}$ & .64 & 0.72 & \\
\hline & Total & .68 & 0.70 & \\
\hline
\end{tabular}

Nota: $* * * p<.001 ; * * p<.01 ; * p<.05$

A la pregunta sobre otros tipos de contenidos responde un $2.4 \%$ de las familias con información que se ha categorizado en: celebraciones (ej. Cumpleaños, $n=1$ ), incumplimiento de normas $(n=1)$, informaciones sobre enfermedades 0 accidentes en el centro ( $n=2)$, sobre las notas $(n=1)$, circulares (ej.: sobre huelgas, $n=2)$, solicitud de tutoría $(n=1)$ o para colaborar en el centro $(n=1)$.

Percepciones de los profesores

Se ofrecen las correlaciones entre las variables cuantitativas consideradas en la muestra de profesorado (Tabla 5). 
Tabla 5

Correlaciones entre las variables cuantitativas de la muestra de profesorado.

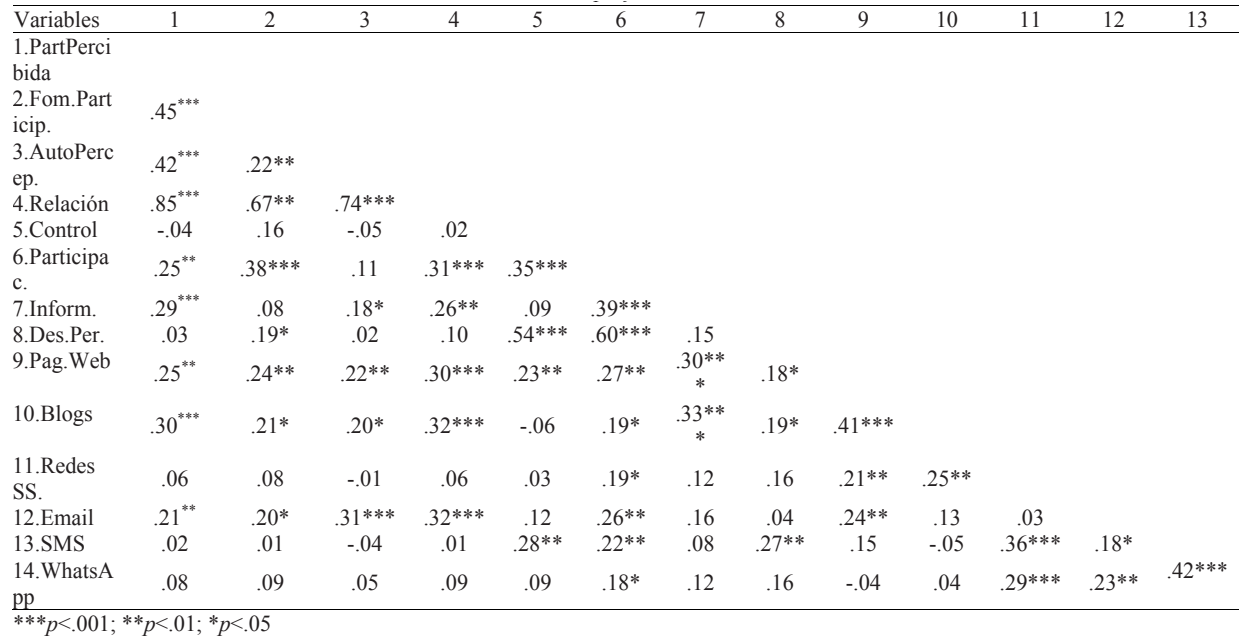

LOS ANOVAS muestran diferencias significativas en la participación percibida, autopercepción de la competencia profesional y en la puntuación global de la escala (Tabla 6), siendo la percepción del profesorado de Secundaria menos positiva (prueba Scheffê). No se detectan, sin embargo, diferencias entre etapas en la variable relativa a la percepción de dificultades con las familias $(F(2,149)=0.20$, n.s.).

Tabla 6

Descriptivos en la muestra del profesorado en la variable relaciones interpersonales.

\begin{tabular}{lllll}
\multicolumn{1}{c}{$\begin{array}{c}\text { Variables de las Relaciones } \\
\text { Interpersonales }\end{array}$} & Factor & Media & DT & Prueba $\mathrm{F}$ \\
\hline Grado de participación & Infantil & 2.54 & 0.80 & \\
& Primaria & 2.29 & 0.77 & $\mathrm{~F}(2,154)=10.22 * * *$ \\
& Secundaria & 1.95 & 0.50 & \\
& Total & 2.22 & 0.72 & \\
\hline Fomento de participación familiar & Infantil & 1.93 & 0.66 & \\
& Primaria & 2.03 & 0.81 & $\mathrm{~F}(2,152)=0.42$ \\
& Secundaria & 1.89 & 0.59 & \\
& Total & 1.95 & 0.69 & \\
\hline Auto-percepción de la competencia & Infantil & 2.96 & 0.51 & \\
profesional & Primaria & 3.07 & 0.64 & $\mathrm{~F}(2,154)=9.59 * * *$ \\
& Secundaria & 2.63 & 0.49 & \\
& Total & 2.87 & 0.59 & \\
\hline Relaciones interpersonales (puntuación & Infantil & 2.54 & 0.52 & \\
global) & Primaria & 2.52 & 0.56 & $\mathrm{~F}(2,154)=9.07 * * *$ \\
& Secundaria & 2.20 & 0.37 & \\
& Total & 2.40 & 0.51 & \\
\hline
\end{tabular}

Nota: $* * * p<.001$ 


\section{LAS tic EN LAS COMUNICACIONES EN LA ESCUELA. PERCEPCIÓN DE PROFESORAdO Y FAMILIAS}

Analizando la percepción del profesorado sobre la idoneidad de las TIC empleadas, se obtienen diferencias significativas en la percepción de los Blogs, siendo menos valorados por el profesorado de Secundaria; y del SMS, que es más valorado en Secundaria que en Infantil (Tabla 7). Por otra parte, el 33.54\% del profesorado informó de emplear la plataforma educativa (e.g. Yedra, Class Dojo; $n=37)$, el teléfono ( $n=7)$, circulares por escrito $(n=6)$ y la agenda escolar $(n=3)$.

Tabla 7

Percepción de las familias del uso y adecuación de las TIC para la comunicación entre la familia y el centro según etapa educativa.

\begin{tabular}{|c|c|c|c|c|c|c|c|}
\hline & \multicolumn{6}{|c|}{ Etapa Educativa } & \multirow[b]{3}{*}{ Prueba F } \\
\hline & \multicolumn{2}{|c|}{ Infantil } & \multicolumn{2}{|c|}{ Primaria } & \multicolumn{2}{|c|}{ Secundaria } & \\
\hline & $\begin{array}{l}\% \mathrm{No} \\
\text { se } \\
\text { utiliza }\end{array}$ & $\begin{array}{c}\text { Media/D } \\
\mathrm{T}\end{array}$ & $\begin{array}{c}\% \mathrm{No} \\
\text { se } \\
\text { utiliza }\end{array}$ & Media/DT & $\begin{array}{l}\% \mathrm{No} \\
\text { se } \\
\text { utiliza }\end{array}$ & Media/DT & \\
\hline $\begin{array}{l}\text { Pág. } \\
\text { web }\end{array}$ & $42.1 \%$ & $\begin{array}{c}3.00 / 0.8 \\
1\end{array}$ & $25.0 \%$ & $2.83 / 0.79$ & $18.0 \%$ & $2.68 / 0.77$ & $\mathrm{~F}(2,113)=1.33$ \\
\hline $\mathrm{B} \log \mathrm{s}$ & $21.1 \%$ & $\begin{array}{c}3.10 / 0.7 \\
2\end{array}$ & $5.5 \%$ & $2.86 / 0.79$ & $33.9 \%$ & $2.31 / 0.86$ & $\begin{array}{l}\mathrm{F}(2,120)=9.41 * \\
* *\end{array}$ \\
\hline $\begin{array}{l}\text { Redes } \\
\text { ss. }\end{array}$ & $64.9 \%$ & $\begin{array}{c}2.17 / 1.0 \\
2\end{array}$ & $59.3 \%$ & $2.61 / 0.84$ & $55.7 \%$ & $2.19 / 1.00$ & $F(2,61)=1.48$ \\
\hline Email & $27.8 \%$ & $\begin{array}{c}2.81 / 0.8 \\
5\end{array}$ & $28.6 \%$ & $3.10 / 0.84$ & $23.3 \%$ & $2.87 / 0.93$ & $F(2,111)=1.10$ \\
\hline SMS & $80.6 \%$ & $\begin{array}{c}1.57 / 0.5 \\
3\end{array}$ & $77.8 \%$ & $2.33 / 1.07$ & $39.0 \%$ & $2.53 / 0.94$ & $\mathrm{~F}(2,54)=3.08 *$ \\
\hline $\begin{array}{l}\text { WhatsA } \\
\mathrm{pp}\end{array}$ & $64.9 \%$ & $\begin{array}{c}2.17 / 1.0 \\
3\end{array}$ & $70.4 \%$ & $2.47 / 1.28$ & $65.0 \%$ & $1.90 / 1.09$ & $F(2,49)=1.15$ \\
\hline Otro/s & $70 \%$ & $\begin{array}{c}3.00 / 1.0 \\
0\end{array}$ & $56.5 \%$ & $3.20 / 0.92$ & $13.3 \%$ & $3.34 / 0.83$ & $F(2,38)=0.29$ \\
\hline
\end{tabular}

Nota: $* * * p<.001, * p=.05$

El análisis de contenido del medio más utilizado para comunicarse con las familias (Tabla 8) identifica al email como el más habitual en todas las etapas, mientras en los primeros niveles educativos los blogs juegan un papel importante, y a partir de Primaria la página web del centro constituye otro canal fundamental de comunicación. Por el contrario, las redes sociales o los mensajes a través del móvil (SMS o WhatsApp) apenas se usan. 
Tabla 8

Porcentaje de la TIC más utilizada para las comunicaciones entre familias y centro según la percepción de las familias (categorías emergentes).

\begin{tabular}{|c|c|c|c|c|c|c|}
\hline & \multicolumn{6}{|c|}{ Etapa educativa } \\
\hline & \multicolumn{2}{|c|}{ Infantil } & \multicolumn{2}{|c|}{ Primaria } & \multicolumn{2}{|c|}{ Secundaria } \\
\hline & $\%$ & Recuento & $\%$ & Recuento & $\%$ & Recuento \\
\hline Blogs & $30.8 \%$ & 12 & $14.0 \%$ & 8 & $0.0 \%$ & 0 \\
\hline Email & $20.5 \%$ & 8 & $28.1 \%$ & 16 & $\begin{array}{c}19.4 \\
\%\end{array}$ & 12 \\
\hline Página web & $7.7 \%$ & 3 & $15.8 \%$ & 9 & $\begin{array}{c}16.1 \\
\%\end{array}$ & 10 \\
\hline Redes sociales & $2.6 \%$ & 1 & $1.8 \%$ & 1 & $0.0 \%$ & 0 \\
\hline WhatsApp & $7.7 \%$ & 3 & $8.8 \%$ & 5 & $1.6 \%$ & 1 \\
\hline SMS & $0.0 \%$ & 0 & $0.0 \%$ & 0 & $8.1 \%$ & 5 \\
\hline Otro/s. & $23.1 \%$ & 9 & $28.1 \%$ & 16 & $\begin{array}{c}53.2 \\
\%\end{array}$ & 33 \\
\hline No contesta & $7.7 \%$ & 3 & $3.5 \%$ & 2 & $1.6 \%$ & 1 \\
\hline
\end{tabular}

Finalmente, se detecta que el profesorado de Infantil y Primaria se comunica más con las familias en temáticas vinculadas con la información y en el caso de Secundaria la mayor atención se dedica al control y seguimiento (Tabla 9). Las pruebas t para muestras relacionadas ( $p<.001$ ) permiten ordenar las temáticas según su tratamiento en las comunicaciones, quedando control y seguimiento e información en primer lugar ( $p=48$ ) seguidos de participación y desarrollo personal ( $p=.77)$, sin diferencias entre dichos pares.

Tabla 9

Descriptivos en las temáticas y resultados de los ANOVAS según etapa educativa del profesorado.

\begin{tabular}{llccc} 
Temáticas de las conversaciones & \multicolumn{1}{c}{ Etapa } & Media & DT & Prueba $F$ \\
\hline \multirow{3}{*}{ Control y Seguimiento } & Infantil & 1.69 & 0.46 & \\
& Primaria & 1.66 & 0.52 & \multirow{2}{*}{$\mathrm{F}(2,150)=19.64 * * *$} \\
& Secundaria & 2.17 & 0.46 & \\
& Total & 1.86 & 0.54 & \\
& Infantil & 1.46 & 0.39 & \\
& Primaria & 1.55 & 0.46 & \multirow{2}{*}{$\mathrm{F}(2,146)=0.48$} \\
& Secundaria & 1.49 & 0.44 & \\
& Total & 1.51 & 0.44 & \\
\hline \multirow{5}{*}{ Información } & Infantil & 2.00 & 0.62 & \\
& Primaria & 2.08 & 0.60 & $\mathrm{~F}(2,149)=6.16^{* *}$ \\
& Secundaria & 1.71 & 0.56 & \\
& Total & 1.92 & 0.61 & \\
\hline \multirow{5}{*}{ Desarrollo personal y adaptación } & Infantil & 1.40 & 0.61 & \\
& Primaria & 1.46 & 0.62 & \multirow{2}{*}{$\mathrm{F}(2,145)=1.24$} \\
& Secundaria & 1.59 & 0.62 & \\
& Total & 1.50 & 0.62 & \\
\hline
\end{tabular}

Nota: $* * * p<.001 ; * * p<.01$ 


\section{LAS TIC EN LAS COMUNICACIONES EN LA ESCUELA. PERCEPCIÓN DE PROFESORADO Y FAMILIAS}

\section{CONCLUSIONES}

Este estudio ha profundizado en la percepción que docentes y familias de distintas etapas educativas tienen de sus relaciones interpersonales en el centro educativo, en cómo valoran el uso de TIC en sus comunicaciones (recursos, idoneidad y temáticas), así como en la relación entre ambas variables, aportando algunas claves sobre el estado de la cuestión en materia de comunicación educativa y TIC.

En cuanto a las relaciones en el centro educativo, al igual que en el estudio de García Sanz y cols. (2010), la percepción de las familias es positiva. Este mismo resultado se encuentra en el profesorado a pesar de que es en la etapa de Secundaria cuando ambos grupos perciben estas relaciones como menos positivas. Respecto a la autopercepción del profesorado sobre su competencia en los procesos de comunicación y gestión de conflictos es en Primaria cuando éste se percibe como más competente. Sin embargo, no se detectan diferencias entre etapas en cuanto a la percepción de dificultades en las relaciones con las familias.

Por lo que se refiere a la percepción de idoneidad de las TIC en las comunicaciones entre familias y centro educativo, tanto profesorado como familias coinciden al percibir más idóneo el uso del SMS en Secundaria que en etapas anteriores; y en que las redes sociales y los mensajes de WhatsApp son los medios menos idóneos. Sin embargo, el medio más empleado sigue siendo el teléfono según las familias, y el email según el profesorado, mientras que los blogs tienen su mayor presencia en Infantil, y a partir de Primaria el canal fundamental de comunicación es la página web del centro y la agenda.

Los contenidos de las comunicaciones mediante las TIC parecen ser tanto para el profesorado como para el alumnado de todas las etapas educativas, fundamentalmente los que se basan en el envío de información sobre temáticas vinculadas con actividades extraescolares, materiales 0 aspectos organizativos y en el control y seguimiento del aprendizaje del alumnado. Los resultados de estudios similares encuentran también que los procesos de comunicación entre el profesorado y familias se centran en los aspectos relacionados con el aprendizaje, aunque en este caso, con los aspectos positivos del mismo (García Sanz y cols., 2010). La atención dedicada al desarrollo personal y social del alumnado, así como a la participación es baja no presentándose diferencias importantes entre etapas educativas al respecto. Como señalan autores como Maciá (2016) "las TIC a día de hoy [...]son en muchas ocasiones meros canales informativos [... ]. Pero, son canales en los que la información fluctúa en una mera dirección, del centro a las familias" (p.81). Apostar por abrir estos canales de información a una auténtica relación bidireccional permitiría ampliar la participación de las familias en los centros educativos mejorando los procesos educativos beneficiando tanto a los docentes como al alumnado y a las familias. La incorporación de las TIC podría servir, si se incorporan usos más participativos y formativos, a disminuir la brecha tecnológica existentes entre las familias como señalan Ballesta y Cerezo (2012) reduciendo la desigualdad entre las diferentes capas sociales y el uso de las nuevas tecnologías (Ricoy, Feliz y Sevillano, 2010).

En cuanto a la vinculación de determinadas temáticas e idoneidad de TIC, Ios resultados muestran que cuanto más idóneas se perciben, más se abordan las temáticas presentadas en el estudio. Por último, se ha demostrado la hipótesis sobre la relación positiva entre la percepción de las relaciones entre la comunidad educativa y la valoración de las TIC, así como con la libertad para abordar diversas temáticas en la muestra del profesorado. En las familias tan sólo la relación con la dirección se vincula con abordar temáticas centradas en la participación y el desarrollo personal, y la relación con el profesorado al uso de blogs. Conviene señalar como limitación al estudio que únicamente la variable sobre las relaciones empleada en la muestra de profesorado atiende a la participación de las familias y a la propia competencia del profesorado para la comunicación.

A la vista de estos resultados se aboga por el fomento de la participación en el centro escolar empleando para ello diversas TIC según la etapa educativa, así como por la promoción de unas relaciones positivas en la comunidad escolar. Al mismo tiempo, se detecta la necesidad de reforzar un uso adecuado de las TIC que conlleve una mejora en la percepción de su idoneidad. Una participación visible y significativa, por su parte, podría también, favorecer el acercamiento y la apertura a temáticas que vayan más allá del control y seguimiento escolar. 


\section{REFERENCIAS BIBLIOGRÁFICAS}

Ballesta, J. y Cerezo, M.C. (2011). Familia y escuela ante la incorporación de las tecnologías del a información y la comunicación. Educación XX1, 14(2),133-156. doi:10.5944/educxx1.14.2.248.

Castro, A. y García, R. (2013). La visión del profesorado de Educación Infantil y Primaria de Cantabria sobre la participación y las relaciones interpersonales entre los miembros de la comunidad escolar. Aula Abierta, 41(1), 73-84.

Diez Ros, R. y Aguilar Hernández, B.M. (2006). Los grupos de WhatsApp escolares: una oportunidad para mejorar la comunicación familia-escuela. En Rosabel Roig-Vila (ed.), Tecnología, innovación e investigación en los procesos de enseñanza-aprendizaje (pp.343-351). Barcelona: Octaedro.

Engel, A.; Coll, C. y Bustos, A. (2010). Aprender y enseñar con tecnologías de la información y la comunicación en la Educación Secundaria. En César Coll (coord.), Desarrollo, aprendizaje y enseñanza en la Educación Secundaria (pp. 105- 130). Barcelona: Graó.

Fernández Enguita, M. y Vázquez Cupeiro, S. (2017). La larga y compleja marcha del clip al clic. Escuela y profesorado ante el nuevo reto digital. Madrid: Fundación Telefónica.

Fernández Prados, J.S. (2012). Los medios de comunicación, nuevas tecnologías de la información y su implicación en el sistema educativo. En Antonio Trinidad Requena (coord.), Sociedad, familia, educación. Una introducción a la Sociología de la Educación (pp. 147- 167). Madrid: Tecnos.

García Sanz, M.P., Gomariz, M.A., Hernández Prados, M.A. y Parra, J. (2010). La comunicación entre la familia y el centro educativo, desde la percepción de los padres y madres de los alumnos. Educatio Siglo XXI, 28(1), 157-188.

Macià, M. (2016). La comunicación familia escuela: el uso de las TIC en los centros de Primaria. Revista Electrónica Interuniversitaria de Formación del Profesorado, 19(1), 73-83. doi:10.6018/reifop.19.1.245841

Macià, M. y Garreta, J. (2018). Accesibilidad y alfabetización digital: barreras para la integración de las TIC en la comunicación familia/escuela. Revista de Investigación Educativa, 36(1), 239-257. doi:10.6018/rie.36.1.290111

Ricoy, M; Feliz, T. y Sevillano, M.L. (2010). Competencias para la utilización de las herramientas digitales en la sociedad de la información. Educación XXI, 13 (1), 199-219.

Ruiz, J. I. (2003). Técnicas de triangulación y control de calidad en la investigación socioeducativa. Bilbao: Universidad de Deusto.

Sánchez-Garrote, I. y Cortada-Pujol, M. (2015). Recursos digitales en la relación familia y escuela en la etapa 03. Cultura y Educación, 27(1), 221-233. doi:10.1080/11356405.2015.1006851

\section{NOTAS}

1 Los datos directos proceden del Informe "Clima escolar, conflicto y gestión de la convivencia en los centros educativos de Cantabria", coordinado por Susana Lázaro-Visa y Andrés A. Fernández-Fuertes (UC) financiado por el Observatorio de la Convivencia Escolar de Cantabria. El Informe puede consultarse en https://www.educantabria.es/docs/planes/convivencia/clima-escolar-conflicto_FN.pdf

2 Considerando municipios rurales aquellos de menos de 2000 habitantes, intermedios de 2000 a 10000 y urbanos de más de 10000 (Instituto Cántabro de Estadística, ICANE). 
